\title{
As competências do professor na educação superior para a aprendizagem dos Millennials e seus sucessores
}

\author{
Rodrigo Freitas, André Guimarães \\ \& Glauco de Menezes
}

Resumo:

Diante dos desafios trazidos pelos Millennials e seus sucessores na educação superior, bem como de um mundo em permanente evolução, considera-se essencial que o professor desenvolva as competências necessárias para que o processo de ensino e aprendizagem dessas gerações seja bem-sucedido. Nesse âmbito, este artigo objetiva identificar quais competências do professor na educação superior são necessárias para a mobilização da aprendizagem dos Millennials e seus sucessores. Consiste em um estudo exploratório, de revisão de literatura, baseado em orientações do método PRISMA (Preferred Reporting Items for Systematic Reviews). Analisa 18 artigos científicos, publicados entre 2007 e 2018 nas bases de dados EBSCOhost, ProQuest, Scopus e Web of Science, que discorrem sobre o tema desta pesquisa. Identifica que os professores na educação superior devem desenvolver oito principais competências: facilitar o processo educativo, estimular o pensamento crítico dos estudantes, utilizar metodologias ativas, desenvolver o uso de tecnologias de informação e comunicação (TICs) em prol da aprendizagem, promover ambiente dinâmico para ensino-aprendizagem, planejar o desenvolvimento da aprendizagem, definir estratégias para avaliação da aprendizagem e aplicar modelo de parceria no processo educacional.

Palavras-chave:

competência do professor; educação superior; Millennials; geração Z. 


\title{
Teacher competencies in higher education for the learning of Millennials and their successors
}

\begin{abstract}
Faced with the challenges brought by the Millennials and their successors in higher education as well as a constantly evolving world, it is essential that the teacher develop the skills necessary for the teaching and learning process of these generations to succeed. In this context, this article aims to identify which competencies of the teacher in higher education are necessary to mobilize the learning of Millennials and their successors. It consists of an exploratory, literature review study based on the PRISMA (Preferred Reporting Items for Systematic Reviews) method. It analyzes 18 scientific papers, published between 2007 and 2018 in the databases EBSCOhost, ProQuest, Scopus and Web of Science, which discuss the theme of this research. It identifies that teachers in higher education should develop eight main competencies: facilitating the educational process, stimulating critical thinking of students, using active methodologies, improving the use of information and communication technologies (ICTs) for learning, promoting a dynamic environment for teaching-learning, planning the development of education, defining strategies for assessment of learning and applying model of partnership in the educational process.
\end{abstract}

Keywords: teacher competency; higher education; millennials; generation Z.

\section{Les compétences du professeur dans l'enseignement supérieur pour l'apprentissage aux Milléniaux et à leurs successeurs}

Résumé: Face aux défis apportés par les Milléniaux et leurs successeurs dans l'enseignement supérieur, tout comme par ceux d'un monde en évolution permanente, on considère comme essentiel que le professeur développe les compétences nécessaires pour que le processus d'enseignement et d'apprentissage à ces générations soit une réussite. Dans ce contexte, cet article a pour objet d'identifier quelles sont les compétences du professeur dans l'enseignement supérieur, nécessaires à la mobilisation de l'apprentissage aux Milléniaux et à leurs successeurs. ॥ s'agit d'une étude exploratoire, d'une revue de la littérature, basée sur les directives de la méthode PRISMA (Preferred Reporting Items for Systematic Reviews). 18 articles scientifiques sont analysés, publiés entre 2007 et 2018 dans les bases de données EBSCOhost, ProQuest, Scopus et Web of Science, qui traitent du thème de cette recherche. ॥ apparaît que les professeurs dans l'enseignement supérieur doivent développer 8 compétences principales: faciliter le processus éducatif, encourager la pensée critique des étudiants, utiliser des méthodologies actives, développer l'utilisation des technologies de l'information et de la communication (TIC) au profit de l'apprentissage, promouvoir un environnement dynamique pour l'enseignement-apprentissage, planifier le développement de l'apprentissage, définir des stratégies pour l'évaluation de l'apprentissage et appliquer un modèle de partenariat dans le processus éducationnel.

Mots-clés: compétence du professeur; enseignement supérieur; Milléniaux; génération Z.

\section{Las competencias del profesor en la educación superior para el aprendizaje de los Millennials y sus sucesores}

Resumen: Ante los desafíos impuestos por los Millennials y sus sucesores en la educación superior, así como de un mundo en permanente evolución, se considera esencial que el profesor desarrolle las competencias necesarias para que el proceso de enseñanza-aprendizaje de esas generaciones tenga éxito. En ese ámbito, este artículo ha tratado de identificar qué competencias del profesor en la educación superior son necesarias para la movilización del aprendizaje de los Millennials y sus sucesores. Por lo tanto, es un estudio exploratorio de revisión de literatura académica basado en orientaciones del método PRISMA (Preferred Reporting Items for Systematic Reviews), en el que se analizan 18 artículos científicos publicados entre 2007 y 2018 en las bases de datos EBSCOHost, ProQuest, Scopus y Web of Science, que tratan sobre el tema de esta investigación. Se destaca que los profesores en la educación superior deben desarrollar ocho competencias principales: facilitar el proceso educativo, estimular el pensamiento crítico de los estudiantes, utilizar metodologías de aprendizaje activo, desarrollar el uso de Tecnologías de la Información y la Comunicación (TIC) en favor del aprendizaje, promover un ambiente dinámico para enseñanzaaprendizaje, planificar el desarrollo del aprendizaje, definir estrategias para la evaluación del aprendizaje y aplicar el modelo de colaboración en el proceso educativo.

Palabras-clave: competencia del profesor; educación superior; generación del milenio; generación Z. 


\section{Introdução}

O atual ambiente de educação superior no século XXI requer professores com perfil distinto daquele que se estabeleceu no passado, de profissionais especialistas em áreas de conhecimento que buscavam renda complementar, status social ou atividade extra às suas jornadas de trabalho (Gil, 2013, pp. 36-37).

A prática para seleção de professores nas instituições de ensino superior brasileiras, que predominava há pouco tempo, era a preferência por sujeitos que demonstravam domínio de conteúdo em alguma matéria e tinham experiência profissional. Assim, o professor ideal era o que ensinava por meio de aulas expositivas sobre temas de seu domínio profissional (Masetto, 2012, p. 15).

Entretanto, o papel de docente do ensino superior nos dias atuais exige uma capacitação própria e específica, que desenvolva a competência pedagógica para o exercício da função de educador, pois cabe ao professor "... a missão de colaborar eficientemente para que seus estudantes aprendam. Esse é seu ofício e compromisso." (Masetto, 2012, p. 15). Portanto, o que se exige atualmente é um professor munido de competências para a prática docente (Gil, 2013, p. 37).

O conceito de competência utilizado por Masetto (2012, p. 15) e Gil (2013, p. 37) advém das proposições de Perrenoud (2000, p. 15): as competências mobilizam, integram e orquestram os recursos cognitivos; a mobilização a que se refere é pertinente ao contexto de cada situação; o exercício da competência passa por esquemas mentais; e as competências profissionais se constroem pela formação e pelas práticas diárias do professor.

Masetto (2012, pp. 13-19) ressalta que a discussão sobre novas exigências e mudanças na prática docente deve considerar fatores como a evolução da estrutura organizacional do ensino superior, o impacto das tecnologias de informação e comunicação (TICS) na criação e compartilhamento de conhecimento e na formação de profissionais, e os requisitos referentes às carreiras profissionais.

Sobretudo, o impacto das TICs coloca o professor em um contexto que suscita muitas perguntas: Como trabalhar com a quantidade de informações que está disponibilizada para todos? Como se manter atualizado com todas as informações existentes? Como subsidiar os estudantes com informações relevantes? Como trabalhar com conteúdos em aula? Como ajudar os estudantes a utilizarem criticamente a Internet? O que se deve ensinar? O que o estudante precisa aprender? Qual o papel do professor? (Masetto, 2012, p. 18).

Em síntese, além do domínio de conhecimento em determinada área, as competências para a prática docente na educação superior propostas por Masetto (2012) abrangem capacidades relacionadas à mediação do processo de aprendizagem, aprimoramento do pensamento crítico dos estudantes, construção coletiva do processo 
de aprendizagem, exploração de novos ambientes de aprendizagem, domínio do uso das TICs, desenvolvimento de processos de avaliação e adoção de técnicas participativas.

A pertinência das reflexões acerca das competências necessárias à prática docente se reafirma perante o perfil atual dos estudantes nas universidades, a maioria pertencente à geração dos Millennials e à Geração Z. Neste sentido, Espinar (2015, p. 100) já afirmara, com base em estudos de diversas instituições, dentre elas a Organisation for Economic Co-operation and Development (OECD), que os estudantes da atualidade constituem a primeira geração de nativos digitais nas universidades.

No Brasil, o censo da educação superior do ano de 2016 revela que a população universitária é constituída majoritariamente por sujeitos na faixa etária dos 18 aos 34 anos, perfazendo $83,19 \%$ do total de matrículas realizadas. Os Millennials representam $63,06 \%$ dos estudantes nos cursos de graduação, enquanto que os pertencentes à Geração Z já correspondem a 20,13\% do total de estudantes matriculados (Instituto Nacional de Estudos e Pesquisas Educacionais Anísio Teixeira, 2017).

Não há dúvida de que os Millennials são mais hábeis em lidar com a tecnologia e possuem diferentes habilidades e experiências, quando comparados às gerações anteriores, e que representam atualmente o perfil dos estudantes dos cursos de graduação (Sandars \& Morrison, 2007, pp. 85-86). A Geração Z, por sua vez, é exposta às mídias digitais em maior intensidade do que a qualquer outro tipo de atividade (Shatto \& Erwin, 2016, p. 253).

Assim, um traço característico dessas gerações é justamente o domínio da tecnologia e a respectiva imersão que fazem nas experiências digitais (Bhana, 2014, p. 1431; Borges \& Vaz, 2013, p. 5; Lemos, 2009, p. 40). Nesse sentido, os Millennials e seus sucessores incorporaram o uso das TICs em suas vidas de tal maneira que é praticamente impossível dissociá-los da tecnologia e imaginá-los vivendo sem a Internet e telefones celulares (Jackson \& Woolsey, 2009, p. 1; Sandars \& Morrison, 2007, p. 85; Thaler, 2013, p. 267).

Dessa forma, esses sujeitos estabeleceram novos padrões de comportamento, tais como: possuem uma relação diferente com o acesso à informação e se distinguem das gerações anteriores pela forma de comunicação com seus pares (Lemos, 2009, p. 45); são permanentemente conectados (Bhana, 2014, p. 1430; Monaco \& Martin, 2007, p. 43; Saleh, Barghuthi, \& Baker, 2017, p. 283; Sandars \& Morrison, 2007, p. 85; Thaler, 2013, p. 267); são colaborativos e trabalham em equipe (Bhana, 2014, p. 1430; Monaco \& Martin, 2007, p. 43; Saleh et al., 2017, p. 283) e são hábeis com multitarefas (Espinar, 2015, p. 116; Monaco \& Martin, 2007, p. 43; Thaler, 2013, p. 268). 
Assim, a mudança do perfil dos estudantes induz uma transformação na postura docente, ou seja, os professores alteram os métodos, hábitos, níveis de cobrança, planejamento e aulas, numa busca de saber lidar, conviver e comunicar com essas novas gerações (Borges \& Vaz, 2013, p. 6). Os métodos convencionais de ensino do passado não mais são eficazes para alcançar os objetivos de aprendizagem com as novas gerações de estudantes (Saleh et al., 2017, p. 283). A adoção de estratégias de aprendizagem multimodais que promovam o engajamento dos estudantes, dentro e fora da escola, pode ser utilizada pelos professores de modo a apoiar o processo de aprendizagem e orientar os estudantes a usarem a tecnologia como ferramenta de aprendizagem, e não somente como entretenimento (Shepherd \& Mullane, 2010, p. 61).

Diante dos desafios trazidos pelos Millennials e seus sucessores, que também são reflexos de um mundo em permanente evolução, urge o esforço para desenvolver as competências mais adequadas para a prática docente na educação superior, privilegiando a centralidade do estudante no processo de mobilização para a aprendizagem, de tal forma que os padrões de comportamento dessas gerações não prejudiquem a eficiência e eficácia do processo de ensino-aprendizagem. Posto isso, o objetivo deste trabalho é identificar as competências do professor na educação superior necessárias para a mobilização da aprendizagem dos Millennials e seus sucessores.

\section{Procedimentos metodológicos}

Esta pesquisa consiste em um estudo exploratório, de revisão de literatura, com abordagem qualitativa e delineamento baseado em pesquisa bibliográfica. Seu desenvolvimento foi realizado a partir de levantamento de artigos científicos, com base no protocolo indicado por Moher et al. (2009), referente ao método PRISMA (Preferred Reporting Items for Systematic Reviews).

O processo de seleção dos artigos em periódicos científicos se constituiu das fases de identificação, triagem, elegibilidade e inclusão dos documentos para a realização da revisão de literatura (Moher et al., 2009).

Para a fase de identificação dos documentos foram definidos os critérios de pesquisa em bases de dados científicas (Tabela 1), sendo que a aplicação da expressão de busca, realizada em 13/06/2018, possibilitou a recuperação de 82 documentos potencialmente elegíveis para a revisão de literatura. 
Tabela 1. Critérios de pesquisa em bases de dados científicas

\begin{tabular}{|c|c|c|c|}
\hline \multirow[t]{2}{*}{ Base de dados } & \multicolumn{2}{|l|}{ Critérios } & \multirow[t]{2}{*}{ Expressão de busca } \\
\hline & Campos pesquisados & Tipos de fontes & \\
\hline EbscoHost & Resumo & $\begin{array}{l}\text { - Revistas acadêmicas } \\
\text { - Periódicos científicos }\end{array}$ & \multirow{4}{*}{$\begin{array}{l}\text { (competence OR ability OR } \\
\text { skill) AND (teacher OR } \\
\text { professor OR faculty) AND } \\
\text { ("high* education" OR } \\
\text { college) AND learning AND } \\
\text { ("millennial" OR } \\
\text { ("generation" AND (y OR z)) } \\
\text { OR ((y OR z) AND } \\
\text { generation)) }\end{array}$} \\
\hline ProQuest & $\begin{array}{l}\text { Qualquer lugar, exceto } \\
\text { texto completo }\end{array}$ & $\begin{array}{l}\text { - Periódicos acadêmicos } \\
\text { - Procedimentos de conferência }\end{array}$ & \\
\hline Scopus & $\begin{array}{l}\text { Título, Resumo, } \\
\text { Palavras-chave }\end{array}$ & $\begin{array}{l}\text { - Journal } \\
\text { - Conference Proceedings }\end{array}$ & \\
\hline Web of Science & $\begin{array}{l}\text { Título, Resumo, } \\
\text { Palavras-chave }\end{array}$ & $\begin{array}{l}\text { - Articles } \\
\text { - Proceedings paper } \\
\text { - Reviews }\end{array}$ & \\
\hline
\end{tabular}

$\mathrm{Na}$ fase de triagem foi realizada a eliminação de itens duplicados (exclusão de 15 documentos) e, por meio da leitura de títulos e resumos, a verificação de aderência do conteúdo dos documentos ao objetivo da presente pesquisa (exclusão de 44 documentos). Assim, 23 documentos publicados no período de 2007 a 2018 foram considerados elegíveis para a leitura do texto completo. Entretanto, os autores obtiveram o acesso ao texto completo de 18 documentos, os quais constituíram o corpus de análise e desenvolvimento da revisão de literatura (Tabela 2).

$\mathrm{Na}$ leitura e análise do texto completo dos artigos do corpus (Tabela 2), procurou-se obter excertos relativos às competências para prática docente junto aos Millennials e seus sucessores. Para tanto, a delimitação deste procedimento de coleta e análise de dados se apoiou nas indicações de competências pedagógicas do professor universitário propostas por Masetto (2012), que foram agrupadas em seis categorias: 1) Mediar o processo de aprendizagem; 2) Aperfeiçoar a capacidade de pensar; 3) Construir um processo coletivo de aprendizagem; 4) Dominar o uso das TICs; 5) Desenvolver o processo de avaliação; e 6) Implantar técnicas participativas. Por fim, o software Atlas.ti foi o recurso tecnológico utilizado para o desenvolvimento dos procedimentos de coleta e análise de dados. 
Tabela 2. Corpus para a revisão de literatura

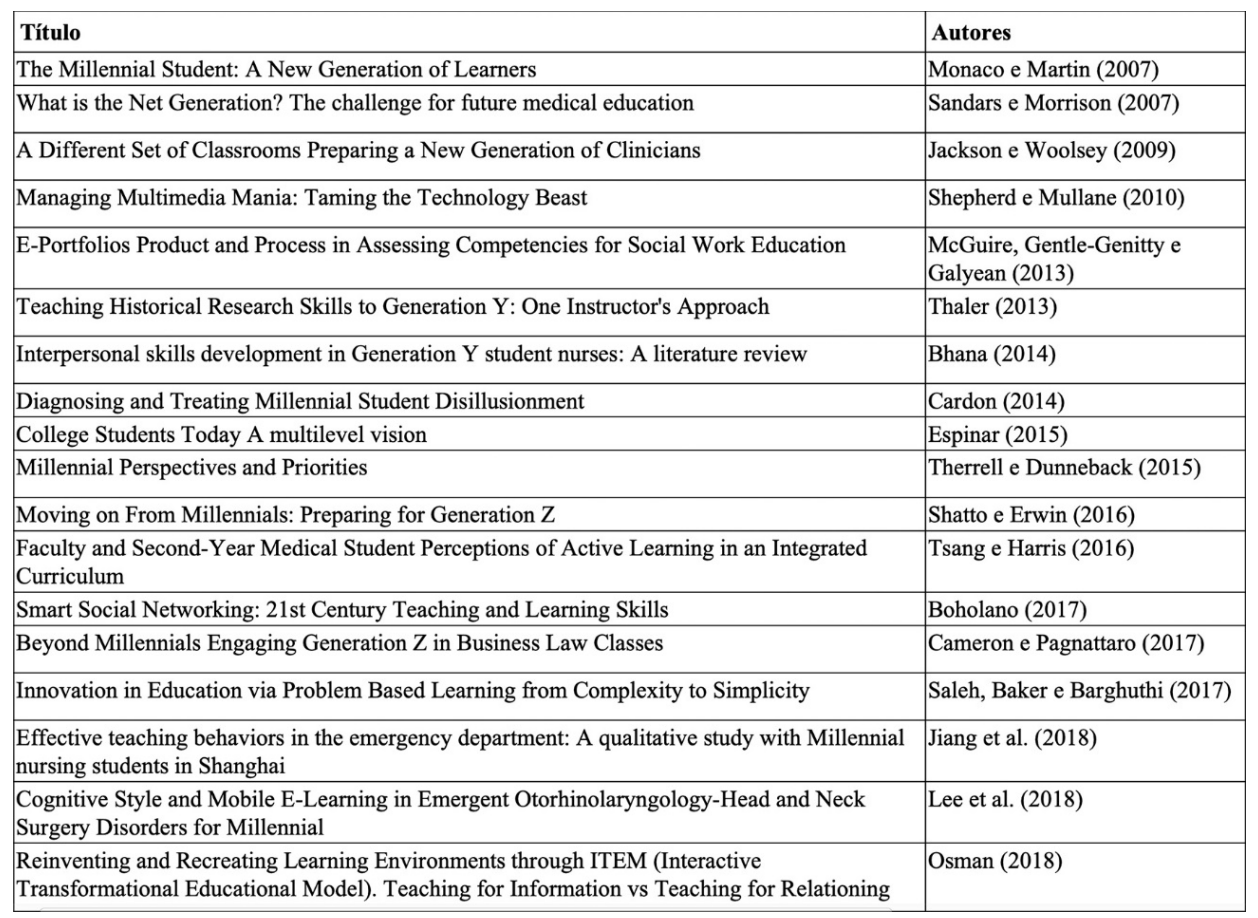




\section{Resultados e discussão}

A Figura 1 apresenta a quantidade de publicações do corpus, por ano, na qual se constata a atualidade do tema com o crescimento da produção científica que aborda o processo de ensino-aprendizagem dos Millennials e seus sucessores a partir de 2013

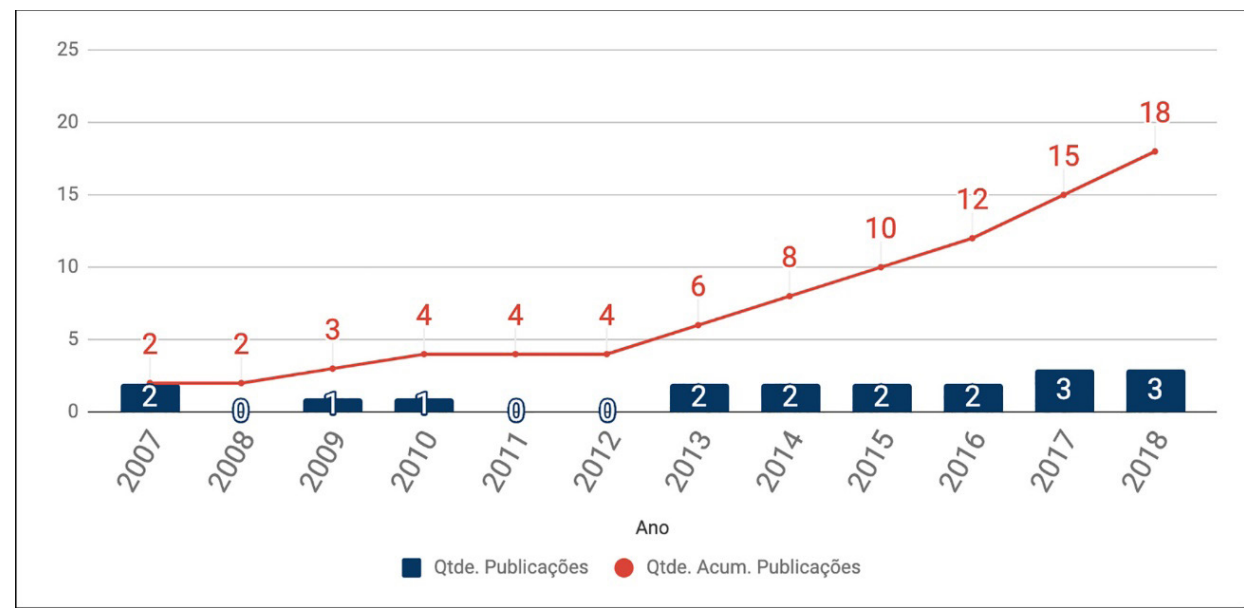

Figura 1. Quantidade de publicações por ano

A análise dos documentos possibilitou identificar oito principais competências do professor na educação superior necessárias para a mobilização da aprendizagem dos Millennials e seus sucessores, sendo que as mais recorrentes foram: utilizar metodologias ativas (citada em 15 artigos - 83\%); promover ambiente dinâmico para ensino-aprendizagem (citada em 14 artigos - 78\%), facilitar o processo educativo (citada em 10 artigos - 56\%) e desenvolver o uso de TICs em prol da aprendizagem (citada em 10 artigos - 56\%).

A Tabela 3 possibilita identificar as competências do professor na educação superior que foram abordadas por cada um dos autores do corpus de pesquisa. 
Freitas, Guimarães \& Menezes: As competências do professor para a aprendizagem dos Millennials

Tabela 3. Competências do professor na Educação Superior, por autores

\begin{tabular}{|c|c|c|c|c|c|c|c|c|}
\hline \multirow[b]{2}{*}{ Autores } & \multicolumn{8}{|c|}{ Competências } \\
\hline & $\begin{array}{c}\text { Facilitar o } \\
\text { processo } \\
\text { educativo }\end{array}$ & $\begin{array}{c}\text { Estimular o } \\
\text { pensamento } \\
\text { crítico dos } \\
\text { estudantes }\end{array}$ & $\begin{array}{c}\text { Utilizar } \\
\text { metodologias } \\
\text { ativas }\end{array}$ & $\begin{array}{c}\text { Desenvolver o } \\
\text { uso de TICs } \\
\text { em prol da } \\
\text { aprendizagem }\end{array}$ & $\begin{array}{c}\text { Promover } \\
\text { ambiente } \\
\text { dinâmico para } \\
\text { ensino- } \\
\text { aprendizagem }\end{array}$ & $\begin{array}{c}\text { Planejar o } \\
\text { desenvolvimento } \\
\text { da aprendizagem }\end{array}$ & $\begin{array}{c}\text { Definir } \\
\text { estratégias para } \\
\text { avaliação da } \\
\text { aprendizagem }\end{array}$ & \begin{tabular}{|c} 
Aplicar \\
modelo de \\
parceria no \\
processo \\
educacional
\end{tabular} \\
\hline Monaco e Martin (2007) & $\checkmark$ & $\checkmark$ & $\checkmark$ & $\checkmark$ & $\checkmark$ & & $\checkmark$ & \\
\hline Sandars e Morrison (2007) & $\checkmark$ & & & $\checkmark$ & $\checkmark$ & & & \\
\hline McGuire, Gentle-Genitty e Galyean (2013) & $\checkmark$ & $\checkmark$ & $\checkmark$ & & $\checkmark$ & $\checkmark$ & & $\checkmark$ \\
\hline Thaler (2013) & & & $\checkmark$ & & & $\checkmark$ & & \\
\hline Bhana (2014) & & $\checkmark$ & $\checkmark$ & & $\checkmark$ & & & \\
\hline Cardon (2014) & $\checkmark$ & & $\checkmark$ & $\checkmark$ & $\checkmark$ & $\checkmark$ & $\checkmark$ & \\
\hline Espinar (2015) & & & $\checkmark$ & & & $\checkmark$ & & $\checkmark$ \\
\hline Boholano (2017) & & & $\checkmark$ & $\checkmark$ & & & & \\
\hline Cameron e Pagnattaro (2017) & & & $\checkmark$ & $\checkmark$ & $\checkmark$ & & & $\checkmark$ \\
\hline Saleh, Baker e Barghuthi (2017) & & & $\checkmark$ & & $\checkmark$ & & & \\
\hline Jiang et al. (2018) & $\checkmark$ & & $\checkmark$ & $\checkmark$ & $\checkmark$ & & & $\checkmark$ \\
\hline Lee et al. (2018) & $\checkmark$ & & $\checkmark$ & $\checkmark$ & $\checkmark$ & & & \\
\hline Osman (2018) & $\checkmark$ & & & & $\checkmark$ & $\checkmark$ & $\checkmark$ & $\checkmark$ \\
\hline
\end{tabular}

Essas competências foram agrupadas em seis categorias, definidas com base em Masetto (2012). Para cada uma das categorias, foram identificadas as suas características, conforme apresentado na Tabela 4.

Tabela 4. Características das competências do professor na educação superior, por categorias

\begin{tabular}{|c|c|c|}
\hline \begin{tabular}{|l|} 
Categorias \\
(MASETTO, 2012)
\end{tabular} & Competências & Características \\
\hline \multirow[t]{2}{*}{$\begin{array}{l}\text { (1) Mediar o processo de } \\
\text { aprendizagem }\end{array}$} & Facilitar o processo educativo & $\begin{array}{l}\text { - Adequação do nível de dificuldade } \\
\text { - Entusiasmo pelo exercício da profissão } \\
\text { - Demonstração de atenção com o aprendiz }\end{array}$ \\
\hline & $\begin{array}{l}\text { Planejar o desenvolvimento da } \\
\text { aprendizagem }\end{array}$ & $\begin{array}{l}\text { - Planejamento dos recursos de ensino-aprendizagem } \\
\text { - Colocação do estudante no centro do processo de aprendizagem } \\
\text { - Sensibilização para mudança }\end{array}$ \\
\hline $\begin{array}{l}\text { (2) Aperfeiçoar a capacidade } \\
\text { de pensar }\end{array}$ & $\begin{array}{l}\text { Estimular o pensamento } \\
\text { crítico dos estudantes }\end{array}$ & $\begin{array}{l}\text { - Desenvolvimento da habilidade de comunicação } \\
\text { - Compreensão profunda das informações recebidas } \\
\text { - Desenvolvimento da crítica: debates, estudos de caso, dramatizações, simulações, práticas de jornalismo. }\end{array}$ \\
\hline \multirow[t]{2}{*}{$\begin{array}{l}\text { (3) Construir um processo } \\
\text { coletivo de aprendizagem }\end{array}$} & $\begin{array}{l}\text { Promover ambiente dinâmico } \\
\text { para ensino-aprendizagem }\end{array}$ & $\begin{array}{l}\text { - Intensificação do uso de TICs } \\
\text { - Engajamento dos estudantes } \\
\text { - Exploração de novos ambientes para a aprendizagem }\end{array}$ \\
\hline & $\begin{array}{l}\text { Aplicar modelo de parceria no } \\
\text { processo educacional }\end{array}$ & $\begin{array}{l}\text { - Promoção da criatividade, confiança, empatia e cuidado entre os integrantes do processo educacional } \\
\text { - Desenvolvimento da autonomia dos estudantes } \\
\text { - Priorização dos relacionamentos }\end{array}$ \\
\hline (4) Dominar o uso das TICs & $\begin{array}{l}\text { Desenvolver o uso das TICs } \\
\text { em prol da aprendizagem }\end{array}$ & $\begin{array}{l}\text { - Orientação do uso adequado das TICs } \\
\text { - Utilização das TICs como suporte e não fim da atividade educacional } \\
\text { - Conhecimento técnico suficiente dos docentes }\end{array}$ \\
\hline $\begin{array}{l}\text { (5) Desenvolver o processo } \\
\text { de avaliação }\end{array}$ & $\begin{array}{l}\text { Definir estratégias para } \\
\text { avaliação da aprendizagem }\end{array}$ & $\begin{array}{l}\text { - Elaboração de planos de ensino } \\
\text { - Realização de avaliação contínua e feedbacks frequentes } \\
\text { - Utilização de rubricas }\end{array}$ \\
\hline $\begin{array}{l}\text { (6) Implantar técnicas } \\
\text { participativas }\end{array}$ & Utilizar metodologias ativas & $\begin{array}{l}\text { - Incentivo à interação e à colaboração } \\
\text { - Atividades baseadas em problemas } \\
\text { - Trabalhos práticos e atividades experienciais }\end{array}$ \\
\hline
\end{tabular}


Na sequência, estão descritas as competências identificadas nesta revisão de literatura, suas características e as principais ideias dos autores pesquisados.

\subsection{Facilitar o processo educativo}

No contexto educacional contemporâneo, os professores devem desempenhar a função de facilitadores da aprendizagem (Monaco \& Martin, 2007, p. 43; Osman, 2018, p. 190; Sandars \& Morrison, 2007, p. 87).

Sandars e Morrison (2007) consideram que cada vez mais são necessários professores com capacidade de facilitar o processo de aprendizagem, e menos daqueles que se ocupam em oferecer conteúdos e informações, pois os estudantes necessitam de habilidades para desenvolver, acessar e avaliar os mais diversos recursos de aprendizagem. Na visão desses autores, os próprios professores constituem a principal barreira para o desempenho da função de facilitação da aprendizagem: “... parece haver relutância entre muitos educadores em se afastar dos métodos tradicionais de ensino e, de fato, existe um senso no qual isto é entendido como uma desistência radical de um sistema que funcionou bem por tão longo tempo." (Sandars \& Morrison, 2007, p. 87, tradução própria). Uma estratégia para lidar com essa relutância é a adoção de novas formas de facilitação da aprendizagem integradas aos programas existentes (Sandars \& Morrison, 2007, p. 87), numa abordagem que combina práticas tradicionais e inovadoras no processo de ensino e aprendizagem.

Therrell e Dunneback (2015, p. 58) destacam quatro fatores da prática docente que impedem a aprendizagem dos estudantes: nível apropriado de desafio; falta de estímulo; falta de paixão ou entusiasmo; e falta de atenção. Isto posto, a função de facilitador da aprendizagem requer dos professores a capacidade de propor atividades que desafiem seus estudantes em um nível adequado de dificuldade, estimulando-os na busca de aprendizado e conhecimento; o entusiasmo pelo exercício de sua profissão e a demonstração de atenção para com seus aprendizes. Em síntese, Therrell e Dunneback (2015, p. 61 tradução própria) afirmam que “... os estudantes parecem querer professores que ensinem com coração, isto é, com paixão, entusiasmo e atenção, que, por sua vez, podem ser práticas vitais para aumentar a motivação deles para aprender".

\subsection{Estimular o pensamento crítico dos estudantes}

Para Monaco e Martin (2007, p. 45), a habilidade de pensamento crítico dos estudantes se desenvolve quando a prática docente se caracteriza pelo incentivo à aprendizagem de conceitos e princípios relacionados à organização do conhecimento, aprimorando a retenção e recuperação de informações. Neste aspecto, práticas como a preparação de textos, cartazes e apresentações orais são fáceis de serem 
implementadas e contribuem para o desenvolvimento do pensamento crítico e construção do conhecimento, pois ajudam no desenvolvimento de habilidades de comunicação e na compreensão mais aprofundada das informações recebidas. A propósito, há a necessidade de incutir nos estudantes o pensamento crítico, a disposição para não se prenderem a instruções passo a passo e a mentalidade de não se contentarem com a facilidade da obtenção de informações imediatas na Internet (Shepherd \& Mullane, 2010, p. 66).

Bhana (2014, p. 1432) corrobora com essa questão afirmando que a necessidade de adoção de estratégias de ensino que apoiem o raciocínio crítico e práticas baseadas em evidências, tendo em vista os estilos de aprendizagem dos Millennials. Dentre estas estratégias, Bhana (2014) considera que debates, estudos de caso, dramatizações, simulações, práticas de jornalismo e a Internet são abordagens que motivam a aprendizagem e oportunizam a análise das informações.

\subsection{Utilizar metodologias ativas}

Nas universidades, os estudantes têm preferido um ambiente de aprendizagem orientado a equipes e de colaboração (Monaco \& Martin, 2007, p. 45). Na visão de Thaler (2013, p. 270), os Millennials reagem melhor em oportunidades de aprendizagem ativa e em atividades que maximizam a interação entre eles, como grupos de discussão ou projetos. Para Saleh, Baker e Barghuthi (2017, p. 283), os Millennials são orientados a equipes e preferem experiências colaborativas e de aprendizagem experiencial.

Em relação às estratégias de aprendizagem ativa, Bhana (2014, p. 1434) destaca a adoção de práticas que promovam o questionamento crítico e o pensamento analítico, como debates, estudos de caso, dramatizações, narrativas, jornalismo e simulações. Outra prática é a aprendizagem baseada em problemas (ABP), que melhora as habilidades de comunicação dos estudantes e pressupõe o trabalho em equipe (Bhana, 2014, p. 1432; Jackson \& Woolsey, 2009, p. 4; Lee et al., 2018, p. 3). Para Saleh, Baker e Barghuthi (2017, p. 283), a ABP representa uma mudança radical da abordagem educacional nas universidades, pois é um processo interativo que impele os estudantes a buscarem conhecimentos necessários para a análise e solução de problemas, que de fato são os direcionadores da aprendizagem.

Therrell e Dunneback (2015, p. 55) identificam que os estudantes preferem trabalhos práticos, laboratórios interativos e atividades experienciais, por promoverem maior envolvimento e atuarem como elementos essenciais para o aprendizado.

Shatto e Erwin (2016, p. 254) destacam o uso de vídeos, mídias sociais e narrativas como ferramentas úteis que promovem engajamento e oportunidades de reflexão. $O$ uso de mídias sociais nas salas de aula possibilita ao professor abordar questões que suscitam reflexões sobre a adequação, confidencialidade e responsabilidade ética no 
uso da Internet. Como ressaltado por Boholano (2017), os professores necessitam incorporar as mídias sociais nas práticas de ensino e aprendizagem, pois a tecnologia é uma ferramenta extraordinária para complementar e aperfeiçoar o ambiente de aprendizagem.

\subsection{Desenvolver o uso das TICs em prol da aprendizagem}

Tanto os Millennials como a Geração Z apresentam domínio e experiência com as TICs (Bhana, 2014; Monaco \& Martin, 2007; Shatto \& Erwin, 2016; Thaler, 2013).

As facilidades de comunicação providas pelas TICs criam oportunidades para os estudantes interagirem entre si. Neste ambiente, o professor deve orientar o processo em prol da aprendizagem, atuando como um facilitador que ensina aos estudantes como aprender, interpretar, aplicar e criticar os temas propostos. Isto é importante em função de que os estudantes têm acesso imediato a muitas informações, mas muitas vezes não têm os instrumentos para usar a tecnologia de modo a obter a informação necessária à construção do pensamento crítico (Monaco \& Martin, 2007, p. 45).

No contexto em que o professor desempenha o papel de facilitador da aprendizagem, as mudanças em relação ao desenvolvimento das competências necessárias à prática docente envolvem uma nova forma de ensinar, na qual a aprendizagem se torna um processo em rede, facilitado pelas TICs. Assim, a aprendizagem passa a depender de como os estudantes conseguem facilmente acessar vários recursos de aprendizagem, tanto os que estão livremente disponibilizados em websites como aqueles que são especificamente criados por educadores para melhorar a aprendizagem, por exemplo, wikis e blogs, tornando a aprendizagem numa experiência mais personalizada para os estudantes (Sandars \& Morrison, 2007, p. 87).

Para tanto, Shepherd e Mullane (2010, pp. 65-66) afirmam que as escolas devem oferecer oportunidades e experiências apropriadas que desenvolvam o conhecimento e a competência dos estudantes na utilização da tecnologia como uma ferramenta para a aprendizagem, e que professores devem deter conhecimentos técnicos suficientes para orientar os estudantes na escolha das ferramentas tecnológicas a serem usadas.

Shatto e Erwin (2016, p. 254) sugerem várias possibilidades de adoção das TICs como recursos para a aprendizagem: uso de tecnologias móveis e aplicativos; uso de leituras que possam ser concluídas em tablets ou smartphones; incentivo à colaboração por meio de redes sociais como o Facebook ${ }^{\circledR}$, Tumblr, Twitter, blogs e grupos de discussão; reforço de conceitos por meio de vídeos disponíveis no Youtube, e jogos interativos como o Kahoot $^{\circledR}$ e Socrative ${ }^{\circledR}$, que tornam a aprendizagem mais atrativa, encorajam o pensamento crítico e possibilitam a realização de feedback.

Boholano (2017, p. 27) ressalta que as tecnologias disponíveis no século XXI são ferramentas extraordinárias para melhorar o ambiente de aprendizagem, e afirma que, 
todavia, as competências digitais são necessárias para o professor garantir que a tecnologia seja usada para suplementar, e não substituir, os métodos instrucionais de alta qualidade.

\subsection{Promover ambiente dinâmico para ensino-aprendizagem}

Um ambiente dinâmico para o ensino-aprendizagem se alcança por meio de iniciativas como a intensificação do uso das TICs e o engajamento dos estudantes. Os avanços promovidos pelas tecnologias possibilitam, por exemplo, a criação de comunidades virtuais de aprendizes, num ambiente dinâmico onde são compartilhados arquivos e recursos de aprendizagem (Sandars \& Morrison, 2007, p. 87).

O engajamento ativo dos estudantes é incentivado quando professores desempenham o papel de facilitadores da aprendizagem (Monaco \& Martin, 2007, p. 43). A aplicação de teoria e prática baseada em evidências de situações concretas, bem como a criação de um ambiente de sala de aula estimulante favorecem o engajamento dos Millennials (Monaco \& Martin, 2007, p. 46; Therrell \& Dunneback, 2015, pp. 55, 59). Assim, as estratégias de ensino devem incluir atividades que possibilitem oportunidades para a participação ativa dos estudantes (Bhana, 2014, p. 1433), por exemplo, a utilização de recursos de multimídia (Shatto \& Erwin, 2016, p. 254) e a aprendizagem baseada em problemas, que promove um ambiente produtivo, pois os Millennials gostam de trabalhar em equipe e preferem atividades colaborativas e experienciais (Saleh et al., 2017, p. 283). Osman (2018, p. 190) corrobora a respeito da importância do engajamento dos estudantes, afirmando que eles são encorajados a se tornarem cocriadores do ambiente de ensino pelo envolvimento no processo educacional, dentro e fora das salas de aula.

\subsection{Planejar o desenvolvimento da aprendizagem}

Atender às expectativas deste perfil emergente de estudantes, tidos como aprendizes impacientes (Cardon, 2014, p. 35; Espinar, 2015, p. 100; Thaler, 2013, p. 271), requer uma mudança de paradigma na estrutura educacional. O planejamento é fundamental para que além dos estudantes, professores e instituições de ensino possam colher os benefícios das metodologias ativas e TICs aplicadas ao processo de aprendizagem (McGuire, Gentle-Genitty, \& Galyean, 2013, p. 108).

Tsang e Harris (2016, p. 452) afirmam que, apesar dos resultados positivos do uso de metodologias ativas, muitos discentes alegam que o tempo disponibilizado não é compatível com as atividades propostas. Por isso, segundo esses autores, a alocação de recursos materiais e de tempo é componente crucial para que os estudantes não constatem a falta de condições adequadas para a realização do projeto pedagógico (Tsang \& Harris, 2016, p. 452). 
Além dos recursos adequados, o planejamento das atividades deve focar no principal interessado: o estudante. Segundo Cardon (2014, p. 39), as adaptações do ensino devem ir além da mudança nas leituras e nas tarefas. Encorajar os estudantes a pensar sobre o uso dos conteúdos abordados nas aulas, em situações futuras, é despertar o interesse pela participação e tornar valioso o tempo em sala (Cardon, 2014, p. 39).

Therrell e Dunneback (2015, p. 254) apresentam algumas sugestões práticas de atividades para os Millennials, como a promoção de discussões sobre inclusão e tolerância, trabalhos em grupos com pontos de vista variados, storytelling, tarefas de laboratório e leituras de acordo com as necessidades de informação.

Independentemente das metodologias adotadas no planejamento, um item não pode ser desconsiderado: a necessidade de mudança. Osman (2018, p. 188) afirma que os docentes devem possuir uma visão consistente e disposição à mudança, e que é necessário encontrar formas de se relacionar, sem resistência, com esta geração emergente de estudantes que está habituada com ferramentas ainda pouco exploradas pelas estruturas educacionais antigas.

\subsection{Definir estratégias para avaliação da aprendizagem}

A elaboração de planos de ensino ou currículos centrados na aprendizagem pode ser útil para prestar feedback aos estudantes. Neles são definidas as tarefas previstas com as respectivas ferramentas de avaliação, proporcionando diretrizes claras para os estudantes. Assim, os feedbacks são fornecidos quando os estudantes realizam as atividades previstas, com comentários ou observações sobre o que foi realizado nas tarefas (Monaco \& Martin, 2007, p. 45).

O estudo conduzido por Therrell e Dunneback (2015, p. 59) indica que, na percepção dos estudantes, um plano de ensino com a programação das atividades clara e detalhada e feedbacks frequentes contribui para que eles sejam bem sucedidos em seus estudos. Ainda, a estratégia de avaliação preferida pelos estudantes consiste na realização de exames ou questionários pouco extensos, mas mais frequentes. Neste aspecto, o argumento apresentado pelos estudantes é que quanto maior o tempo entre os exames, maior a quantidade de informações que eles teriam que memorizar. Para Therrell e Dunneback (2015, p. 59), isto indica que os estudantes desejam explorar os conteúdos e aprender mais, em vez de simplesmente memorizar informações para a realização de exames.

Osman (2018, p. 190) relata que avaliações contínuas e feedbacks frequentes são estratégias adotadas em plataformas interativas ou ambientes virtuais de aprendizagem que envolvem diretamente os estudantes no processo de avaliação da aprendizagem.

Outro instrumento útil para a avaliação da aprendizagem é a elaboração de rubricas. Elas explicitam as expectativas dos professores, mas também propiciam aos 
estudantes o controle direto sobre o que se almeja no processo de aprendizagem. Neste caso, o desafio para os professores é desenvolver rubricas que sejam claras e justas, visto que a questão principal diz respeito a determinar o que torna uma avaliação válida e a como desenvolver e gerenciar rubricas que direcionem o sucesso dos estudantes (Shepherd \& Mullane, 2010, p. 66).

\subsection{Aplicar modelo de parceria no processo educacional}

O modelo de parceria no processo educacional é explicado pelo cenário no qual os estudantes deixam de ser receptores passivos do conhecimento transferido pelo professor e passam a trabalhar ativamente junto ao docente, que se torna um facilitador no processo de aquisição de informação (Osman, 2018, p. 189). Quando o professor deixa a posição dominante de possuidor absoluto do conhecimento para fomentar a parceria com os estudantes, há a promoção de criatividade, confiança, empatia e cuidado entres os integrantes do processo educacional, fazendo com que os estudantes se tornem cocriadores do ambiente de aprendizagem (Osman, 2018, pp. 189-190).

O cuidado dos professores ajuda a desenvolver a autonomia dos Millennials, proporcionando um ambiente de respeito mútuo, propício ao bem-estar dos estudantes que eleva sua sensação de pertencimento e, por consequência, melhora sua aprendizagem (Jiang et al., 2018, p. 224). A relação de harmonia entre corpo docente e discente é um importante elemento na dinâmica da sala de aula (Cameron \& Pagnattaro, 2017, p. 323; Jiang et al., 2018, p. 224).

Osman (2018, pp. 189-190) aponta a adoção do modelo de parceria como a única chance de progresso em nossa cultura, seja no âmbito educacional ou no âmbito social. Buscar o modelo de parceria é fortalecer as relações. Na educação, este modelo coloca o aprendiz no centro das atenções, que desde a infância é motivado a aceitar, apreciar, respeitar e valorizar os sentimentos de todos. Quando o estudante se torna o centro do processo de ensino, a interação pedagógica apresenta uma nova perspectiva onde os relacionamentos tornam-se fundamentais, na qual se incentiva o envolvimento do estudante dentro e fora da sala de aula e a parceria com o professor, como facilitador da educação. Todos possuem a oportunidade de vivenciar valores como tolerância, compaixão, força e liderança.

\section{Considerações finais}

A profissão de professor na educação superior está mudando e as demandas do século XXI exigem, além de conhecimento técnico, competências que antes eram colocadas em segundo plano. Com a predominância dos Millennials nas instituições de ensino superior, torna-se indispensável promover estratégias de aprendizagem direcionadas a este novo perfil de aluno. 
Assim, a partir da análise de um corpus constituído de 18 artigos científicos, foram identificadas oito competências do professor na educação superior para a aprendizagem dos Millennials e seus sucessores: facilitar o processo educativo, estimular o pensamento crítico dos estudantes, utilizar metodologias ativas, desenvolver o uso das TICs em prol da aprendizagem, promover ambiente dinâmico para ensino-aprendizagem, planejar o desenvolvimento da aprendizagem, definir estratégias para avaliação da aprendizagem e aplicar modelo de parceria no processo educacional.

Dentre estas competências, ressalta-se que as TICs são ferramentas fundamentais para a promoção de um ambiente dinâmico para ensino-aprendizagem, que potencializam o uso de metodologias ativas para desenvolver uma aprendizagem em consonância com as características dos Millennials e seus sucessores.

Tais características, que em muito diferem estes novos estudantes de seus antecessores, fazem do ensino superior um cenário complexo e multifacetado. Este trabalho destaca, essencialmente, a importância de o professor buscar uma formação contínua que contribua no desenvolvimento de suas habilidades para a docência neste cenário, tanto no domínio das competências identificadas neste estudo como para se preparar para os desafios de um mundo em permanente evolução.

Contudo, é fundamental ressaltar que tais considerações são oriundas deste recorte da revisão da literatura, apresentando uma visão global sobre as competências do professor na educação superior para a aprendizagem dos Millennials e seus sucessores. O desenvolvimento de uma análise do fenômeno estudado em relação a contextos específicos não integrou os objetivos deste trabalho, a despeito do corpus relatar pesquisas desenvolvidas em diferentes localidades e culturas. Assim, para trabalhos futuros sugere-se o desenvolvimento de estudos que relacionem a competência docente perante desafios e aspectos culturais, em contextos de áreas distintas do conhecimento.

\section{Agradecimentos}

O presente trabalho foi realizado com apoio da Coordenação de Aperfeiçoamento de Pessoal de Nível Superior - Brasil (CAPES) - Código de Financiamento 001.

\section{Referências}

Bhana, V. M. (2014). Interpersonal skills development in Generation Y student nurses: A literature review. Nurse Education Today, 34(12), 1430-1434. https://doi.org/10.1016/j.nedt.2014.05.002.

Boholano, H. B. (2017). Smart Social Networking: 21st Century Teaching and Learning Skills. Research in Pedagogy, 7(1), 21-29.

Borges, B. S., \& Vaz, L. R. (2013). Mudanças impostas pela geração "Y" nos contextos político e educacional brasileiro. Cadernos Da FUCAMP, 12(16), 1-11. 
Freitas, Guimarães \& Menezes: As competências do professor para a aprendizagem dos Millennials

Cameron, E. A., \& Pagnattaro, M. A. (2017). Beyond Millennials: Engaging Generation Z in Business Law Classes. Journal of Legal Studies Education, 34(2), 317-324.

Cardon, L. S. (2014). Diagnosing and Treating Millennial Student Disillusionment. Change: The Magazine of Higher Learning, 46(6), 34-40.

Espinar, S. R. (2015). College Students Today: A multilevel vision. Redu-Revista De Docencia Universitaria, 13(2), 91-124.

Gil, A. C. (2013). Didática do ensino superior (1. ed.). São Paulo: Atlas.

Instituto Nacional de Estudos e Pesquisas Educacionais Anísio Teixeira. (2017). Sinopse Estatística da Educação Superior 2016. Retrieved June 23, 2018, from http://portal.inep.gov.br/web/guest/ sinopses-estatisticas-da-educacao-superior.

Jackson, C. A., \& Woolsey, J. D. (2009). A Different Set of Classrooms: Preparing a New Generation of Clinicians. Forum on Public Policy Online, 2009(1), 0-9.

Jiang, J., Zeng, L., Kue, J., Li, H., Shi, Y., \& Chen, C. (2018). Effective teaching behaviors in the emergency department: A qualitative study with Millennial nursing students in Shanghai. Nurse Education Today, 61, 220-224. https://doi.org/10.1016/j.nedt.2017.12.007.

Lee, L. A., Chao, Y. P., Huang, C. G., Fang, J. T., Wang, S. L., Chuang, C. K., Kang, C. J., Hsin, L. J., Lin, W. N., Fang, T. J., \& Li, H. Y. (2018). Cognitive Style and Mobile E-Learning in Emergent Otorhinolaryngology-Head and Neck Surgery Disorders for Millennial Undergraduate Medical Students: Randomized Controlled Trial. Journal of Medical Internet Research, 20(2).

Lemos, S. (2009). Nativos digitais x aprendizagens: um desafio para a escola. Boletim Técnico Do Senac, 35(3), 38-47.

Masetto, M. T. (2012). Competência pedagógica do professor universitário (2. ed. rev). São Paulo: Summus.

McGuire, L. E., Gentle-Genitty, C., \& Galyean, E. (2013). E-Portfolios: Product and Process in Assessing Competencies for Social Work Education. The Journal of Baccalaureate Social Work, 18, 95-112.

Moher, D., Liberati, A., Tetzlaff, J., Altman, D. G., \& The PRISMA Group (2009). Reprint-Preferred Reporting Items for Systematic Reviews and Meta-Analyses: The PRISMA Statement. Physical Therapy, 89(9), 873-880.

Monaco, M., \& Martin, M. (2007). The Millennial Student: A New Generation of Learners. Athletic Training Education Journal, 2(2), 42-46.

Osman, S. (2018). Reinventing and Recreating Learning Environments through ITEM (Interactive Transformational Educational Model). Teaching for Information vs Teaching for Relationing. In Conference proceedings of eLearning and Software for Education (14th ed., Vol. 1, pp. 187-194). Bucharest. http://doi.org/10.12753/2066-026X-18-026.

Perrenoud, P. (2000). Dez novas competências para ensinar. Porto Alegre: Artmed.

Saleh, M., Barghuthi, N. Al, \& Baker, S. (2017). Innovation in Education via Problem Based Learning from Complexity to Simplicity. In 2017 International Conference on New Trends in Computing Sciences (pp. 283-288). Amman, Jordan. http://doi.org/10.1109/ICTCS.2017.51. 
Sandars, J., \& Morrison, C. (2007). What is the Net Generation? The challenge for future medical education. Medical Teacher, 29(2/3), 85-88.

Shatto, B., \& Erwin, K. (2016). Moving on From Millennials: Preparing for Generation Z. Journal of Continuing Education in Nursing, 47(6), 253-254. https://doi.org/10.3928/00220124-20160518-05.

Shepherd, C., \& Mullane, A. M. (2010). Managing Multimedia Mania: Taming the Technology Beast. Journal of College Teaching \& Learning, 7(1), 59-70.

Thaler, V. S. (2013). Teaching Historical Research Skills to Generation Y: One Instructor's Approach. History Teacher, 46(2), 267-281.

Therrell, J. A., \& Dunneback, S. K. (2015). Millennial Perspectives and Priorities. Journal of the Scholarship of Teaching and Learning, 15(5), 49-63.

Tsang, A., \& Harris, D. M. (2016). Faculty and Second-Year Medical Student Perceptions of Active Learning in an Integrated Curriculum. Advances in Physiology Education, 40(4), 446-453.

Rodrigo de Castro Freitas

Programa de Pós-Graduação em Gestão da Informação

Universidade Federal do Paraná, Brasil

Email: decastro_rf@hotmail.com

ORCID: https://orcid.org/0000-0001-9427-793X

André José Ribeiro Guimarães

Programa de Pós-Graduação em Gestão da Informação

Universidade Federal do Paraná, Brasil

Email: andrejg@gmail.com

ORCID: https://orcid.org/0000-0003-0874-7400

Glauco Gomes de Menezes

Doutor em Educação

Programa de Pós-Graduação em Gestão da Informação

Universidade Federal do Paraná, Brasil

Email: glaucogm@gmail.com

ORCID : https://orcid.org/0000-0003-0445-5646

Correspondência:

Rodrigo de Castro Freitas

Av. Prefeito Lothário Meissner, 632

80.210-170 - Curitiba, PR - Brasil

Data de submissão: Dezembro 2018

Data de avaliação: Março 2019

Data de publicação: Setembro 2019 